\title{
Impact of Microwave Treatment on Chemical Constituents in Fresh Rhizoma Gastrodiae (Tianma) by UPLC-MS Analysis
}

\author{
Qimeng Fan, ${ }^{1}$ Chaoyin Chen, ${ }^{2}$ Dingqi Xie, ${ }^{1}$ and Shenglan Zhao ${ }^{1}$ \\ ${ }^{1}$ Faculty of Chinese Traditional Medicine, Yunnan University of Traditional Chinese Medicine, Yunnan 650500, China \\ ${ }^{2}$ Faculty of Life Science and Technology, Kunming University of Science and Technology, Yunnan 650500, China \\ Correspondence should be addressed to Shenglan Zhao; zhaoshenglan@163.com
}

Received 5 April 2014; Accepted 20 August 2014; Published 14 October 2014

Academic Editor: Dimitris P. Makris

Copyright (C) 2014 Qimeng Fan et al. This is an open access article distributed under the Creative Commons Attribution License, which permits unrestricted use, distribution, and reproduction in any medium, provided the original work is properly cited.

\begin{abstract}
Fresh Rhizoma Gastrodiae (Tianma) was processed in a microwave oven at $2450 \mathrm{MHz}$ in order to study the effect on the main chemical component changes taking place during microwave treatment. It was found that microwave affected the chemical composition of Tianma. Seven compounds, including gastrodin, gastrodigenin (p-hydroxybenzylalcohol), phydroxybenzaldehyde, vanillyl alcohol, vanillin, adenine, and 5-hydroxymethylfurfural, were identified in this study. As major active compounds, the contents of gastrodin and gastrodigenin in MWT Tianma were both twice as much as those in raw Tianma. Besides, the MS data show that there are still some unidentified compositions in Tianma, and there are also many converted compounds in MWT Tianma, which is worthy of further work. The results have indicated that microwave treated fresh Tianma might be helpful in designing the processing of traditional Chinese medicine and the application of microwave technology in traditional Chinese medicine needs to be researched further in the future.
\end{abstract}

\section{Introduction}

Tianma, a dry tuber of Gastrodia elata Blume [1] (Orchidaceae, recorded by Shen Nong's Materia Medica, A.D. 102200, collected in Chinese Pharmacopoeia [2]), is one of the earliest and most important traditional herbal drugs used for thousands of years in the history of traditional Chinese medicine (TCM). In recent years, it has been widely accepted as a health supplement in many countries owing to its remarkable and reliable medical benefits. According to the theory of TCM, Tianma is sweet in taste and peaceful in property and enters the liver and liver meridians. Phytochemical investigation has found that the components of Tianma include gastrodin ( $\mathrm{p}$-hydroxymethylphenyl- $\beta$-Dglucopyranoside), gastrodigenin (p-hydroxybenzylalcohol), vanillyl alcohol, parishin, gastrodioside, vanillin, and glycoproteins. Among them, gastrodin and gastrodigenin are considered the major active components [3]. Due to those physiological substances, Tianma and its extract products are used as an anticonvulsant, an analgesic, and a sedative against general paralysis, epilepsy, vertigo, and tetanus [4].
Microwave is a nonionizing electromagnetic energy at frequencies ranging from $300 \mathrm{MHz}$ to $300 \mathrm{GHz}$ with a wavelength of $1 \mathrm{~m}-1 \mathrm{~mm}$ that causes molecular motion by migration of ions and rotation of dipoles [5]. Due to the electric field, dipole rotation refers to the alignment of molecules that have either permanent or induced dipole moments. Thermal disorder, as the field intensity decreases, is restored which results in thermal energy being released. The absorption of microwave energy and its release as heat strongly depend on the relative permittivity and the dipolar moment of the medium. The greater the relative permittivity is, the more thermal energy is released and the more rapid the heating for a given frequency is. As a consequence, this form of nonionizing electromagnetic energy is transmitted as waves, which can penetrate in biomaterials and interact with polar molecules into materials, such as water to generate heat [6]. In addition to thermal effect, nonthermal effect of microwave (also called not purely thermal and specific microwave effects) cannot be ignored [7]. Recently, there are many studies with regard to the nonthermal effect of microwave [8-10]. The issue of nonthermal effects, however, is still a 
controversial matter. Due to these properties, microwave, as a rapidly developing technology, has been applied in many fields including organic synthesis [11-13], chemical and food drying [14-16], and extraction [17-20].

There are many studies [14-21] concerning microwave on plants, herbs, or their products, most of which focused on microwave assisted extraction and microwave drying or heating. The application of microwave is performed at frequencies $915 \mathrm{MHz}$ at industrial scale and $2450 \mathrm{MHz}$ in domestic ovens [22]. And at the frequency $2450 \mathrm{MHz}$, the alignment of the molecules followed by return to disorder occurs $4.9 \times 10^{9}$ times per second, resulting in rapid heating. Thus, fast heating is the main advantage of microwaves. Obtained from these studies concerning rice, Kaasova et al. reported that the physical and chemical properties such as the viscosity, elasticity, hydrophobicity, damage starch, total starch, and water absorption capacity were not significantly changed during microwave heating as revealed by the graphic characteristics [23]. Recently, Tochampa et al. [24] have found that microwave heating has no adverse effects on chemical, microbiological, and sensory properties of sweet fermented glutinous rice. Thus, it can be seen that these studies mentioned above suggest that microwave has good effects on these matters.

Traditional Chinese medicine including Tianma contains complicated components, which would bring compositional, pharmacological, and functional changes under the effect of external force. Thus, it is worth taking advantage of effects of microwave to study herbal medicines. In the present study, the effect of microwave treatment on fresh Tianma, which was evaluated by changes of several main components, was reported. The aim of this work is to make a preliminary study of microwave treatment on fresh Tianma. This information might be essential for the further study of microwave on Tianma and other herbal medicines.

\section{Experimental}

2.1. Chemicals and Reagents. Acetonitrile (HPLC grade) were procured from Merck \& Co., Inc. (Darmstadt, Germany). Deionized water was purified by Milli-Q system (Millipore, Bedford, MA, USA). Reference standard of gastrodin and gastrodigenin was from Xian Kailai Biological Engineering Co., Ltd. (Xian, PR China), and their purities were all $>98 \%$. Other chemicals and reagents used were of analytical grade and were obtained from Tianjin Fengchuan Chemical Reagent Technologies Co., Ltd. (Tianjin, China).

Fresh Tianma was purchased from the local markets. This Tianma was identified according to the Chinese Pharmacopoeia [2] by Professor Ronghua Zhao (Yunnan University of Traditional Chinese Medicine).

2.2. Apparatus and Chromatographic Conditions. An electric juicer (Joyoung Limited by Share Ltd, Jinan, China) was used to mash up fresh Tianma into a smooth paste. A microwave oven (Guangdong Midea Holding Co., Ltd, Foshan, China) with frequency of $2450 \mathrm{MHz}$ and inner volume of $20 \mathrm{~L}$ was used for microwave processing of fresh Tianma paste. Electronic balance (Ohaus Corporation, Shanghai, China) was used for weighing accurately. Ultrasonic cleaner (Shanghai Kudos Ultrasonic instrument Co., Ltd, Shanghai, China) was applied in the process of solvent extraction.

Chromatographic separation was performed on a Waters Acquity UPLC (Waters, Milford, MA) coupled to Micromass Q-TOF Micro MS (Waters, Milford, MA). An Agilent TCC18(2) Column $(4.6 \mathrm{~mm} \times 250 \mathrm{~mm}, 5 \mu \mathrm{m})$ was used for separation. The elution was carried out at flow rate of $1 \mathrm{~mL} / \mathrm{min}$ using acetonitrile (mobile phase A): $0.1 \%$ formic acid (mobile phase B) mobile phase with detection at $270 \mathrm{~nm}$. The mobile phases were filtered through a $0.22 \mu \mathrm{m}$ membrane filter and degassed. The injection volume was $10 \mu \mathrm{L}$ and analysis was performed at $25^{\circ} \mathrm{C}$. The solvent gradient elution program was as follows: $0-20 \mathrm{~min}, 3 \% \mathrm{~A} ; 20-50 \mathrm{~min}$, linear gradient from 3 to $20 \% \mathrm{~A} ; 50-90 \mathrm{~min}$, linear gradient from 20 to $100 \% \mathrm{~A}$; and $90-95 \mathrm{~min}, 100 \% \mathrm{~A}$ isocratic.

The mass spectrometer was used with the regular ESI interface and calibrated prior to experiments. The mass spectra were recorded with full scan mode in both negative mode and positive mode, and parameters of the two modes are the same. Electrospray ionization interface parameter was as follows: spray voltage: $4.0 \mathrm{kV}$; cone voltage: $30 \mathrm{~V}$; drying gas $\left(\mathrm{N}_{2}\right)$ flow rate: $400 \mathrm{~L} / \mathrm{h}$; capillary heater temperature: $350^{\circ} \mathrm{C}$; and ion source temperature: $110^{\circ} \mathrm{C}$. Data were collected in continuum mode between 100 and $1000 \mathrm{~m} / z$, and the ion current (TIC): $[\mathrm{M}-\mathrm{H}]^{-}$was extracted.

\subsection{Microwave Treatment (MWT) and Preparation of Sample} Solution. A certain amount of fresh Tianma with good condition was chosen to be mashed up into a smooth paste by an electric juicer. Several servings of $20.0 \mathrm{~g}$ of fresh Tianma paste were weighed accurately. Six servings of fresh Tianma paste were differentially treated by microwave for $30 \mathrm{~min}$ at power of $700 \mathrm{~W}$. And leave six samples with no microwave processing.

After the microwave processing, let cool to room temperature. $50 \mathrm{~mL}$ of $70 \%$ methanol was measured accurately and transferred to all the samples including samples with no microwave processing. Total weight of each sample including the weights of fresh Tianma paste, beaker flask, and methanol was weighed accurately and recorded. After the microwave processing, let the microwave treated fresh Tianma cool to room temperature. The supernatants were filtered through $0.45 \mu \mathrm{m}$ membrane filters and degassed, which were considered as the sample solutions.

\section{Result and Discussion}

3.1. Chemical Components of Tianma before and after MWT. Tianma contains a variety of constituents that have previously been characterized. Most published accounts report results of structure identification work on constituents found in either methanolic or aqueous methanolic extracts of rhizome that has been steamed and dried (including products from commercial sources) or directly extracted from the fresh Tianma, followed by chromatographic separation. Recent studies have confirmed the presence of the phenolic glucoside gastrodin and its aglycone gastrodigenin (p-hydroxybenzylalcohol) as primary constituents. Many structurally related constituents 


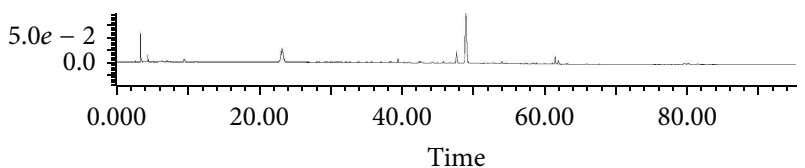

(a)

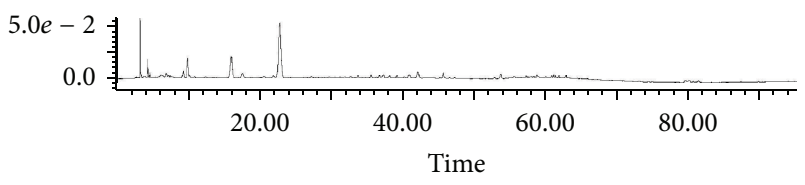

(b)

FIgURE 1: The global chromatograms of (a) raw Tianma extract and (b) MWT Tianma extract.

that are present in smaller amounts continue to be identified; see Zhao et al. [25]. Biological and biochemical activities of several constituents of Tianma are reported in a variety of assays. Gastrodin is the phenolic glucoside of phydroxybenzylalcohol. Closely related constituents, including p-hydroxybenzaldehyde, vanillyl alcohol, and vanillin, have been reported. Recent pharmaceutical researches show that gastrodin and gastrodigenin are the main active chemical constituents of Tianma. Therefore, these components in Tianma would be the focus of this research.

In reversed phase ultraperformance liquid chromatography-electrospray ionization mass spectrometry, both positive and negative ionization modes under optimized operation conditions were full scanned to detect the significant molecular ion peaks of chromatographic peaks with different ionization mode and then determine their molecular weight.

From Figures 1 and 2, compositions of Tianma before and after MWT have been significantly changed under the effect of microwave. There are large differences in chromatograms from 9 to $25 \mathrm{~min}$ and from 40 to $45 \mathrm{~min}$ between raw Tianma extract and MWT Tianma extract.

The representative phenolics of Tianma, including p-hydroxybenzylalcohol, p-hydroxybenzaldehyde, vanillyl alcohol, and vanillin, were identified by UPLC-MS with ESI source in negative mode (Figure 3). Consistent with reference [26] these phenolics exhibited their quasimolecular ions of $[\mathrm{M}-\mathrm{H}]^{-}$for molecule mass information. By extracting the positive ion current, attentive study of the mass spectra of compounds, and comparison with reference data and some standards, gastrodin was identified by UPLC-MS with ESI source in negative mode.

Besides, there are several chemical components whose contents showed a great difference before and after MWT and five chromatogram peaks of which with good response were chosen for analysis. 10 chromatographic peaks were selected and numbered according to their retention time (Figure 2).

From the total ion chromatogram of methanol extract of Tianma, the mass spectrum under both positive and negative ionization modes has good responses. The four phenolics of Tianma were identified in both raw Tianma extract and MWT Tianma extract, (5p-hydroxybenzylalcohol (MW 124), (6)vanillyl alcohol (MW 154), (7) p-hydroxybenzaldehyde

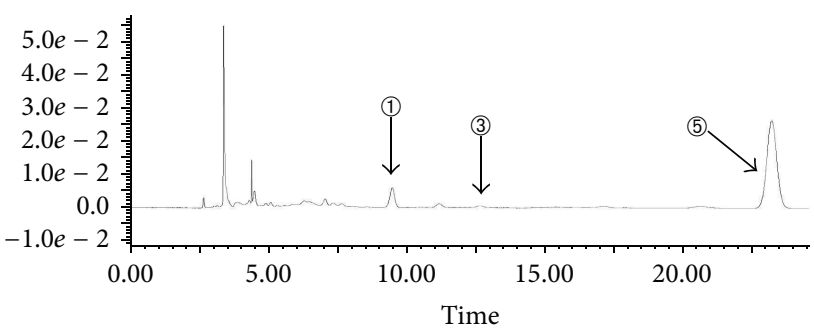

(a)

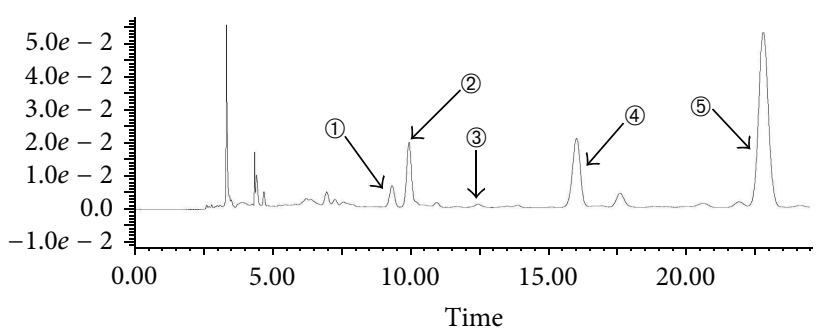

(b)

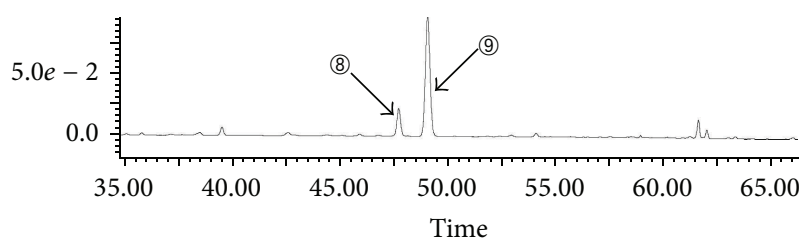

(c)

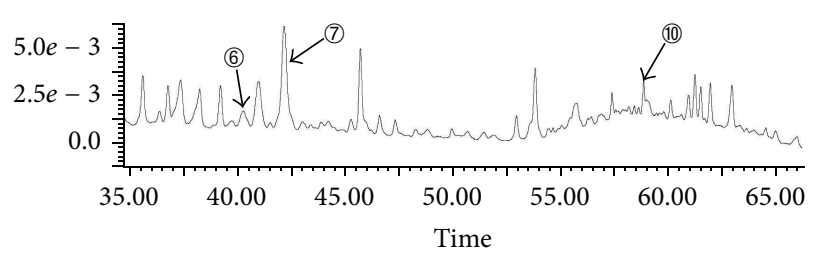

(d)

Figure 2: Partial chromatograms of (a), (c) raw Tianma extract, (b), (d) MWT Tianma extract.

(MW 122), and (10vanillin (MW 152). At the same time, (3) gastrodin, an active component in Tianma, and (1)adenine [27] and (4)5-hydroxymethylfurfural [28] were also identified with an ESI source in positive mode. MS data for chemical components in Tianma before and after MWT are shown in Table 1, and the representative MS spectra are shown in Figure 4.

However, compounds (2), (8), and (9) have not been identified and there were no corresponding reference data in previous research. Through the analysis to their mass spectrum (Figure 5), the possible molecular weights and structures of the three compounds were given in Figure 6. From mass spectrum, compound (9), must contain a benzene circle and a nitrogen atom, and its molecular weight may be 107 from the possible quasimolecular ion $[\mathrm{M}+\mathrm{H}]^{+}(108)$, and the molecular formula may be $\mathrm{C}_{7} \mathrm{H}_{9} \mathrm{~N}$. From mass spectrum and compositions transformation in this study, compound (8) contains the structure of compound (2) from the shared ion peak (213), and molecular weight of (2) and (8) 


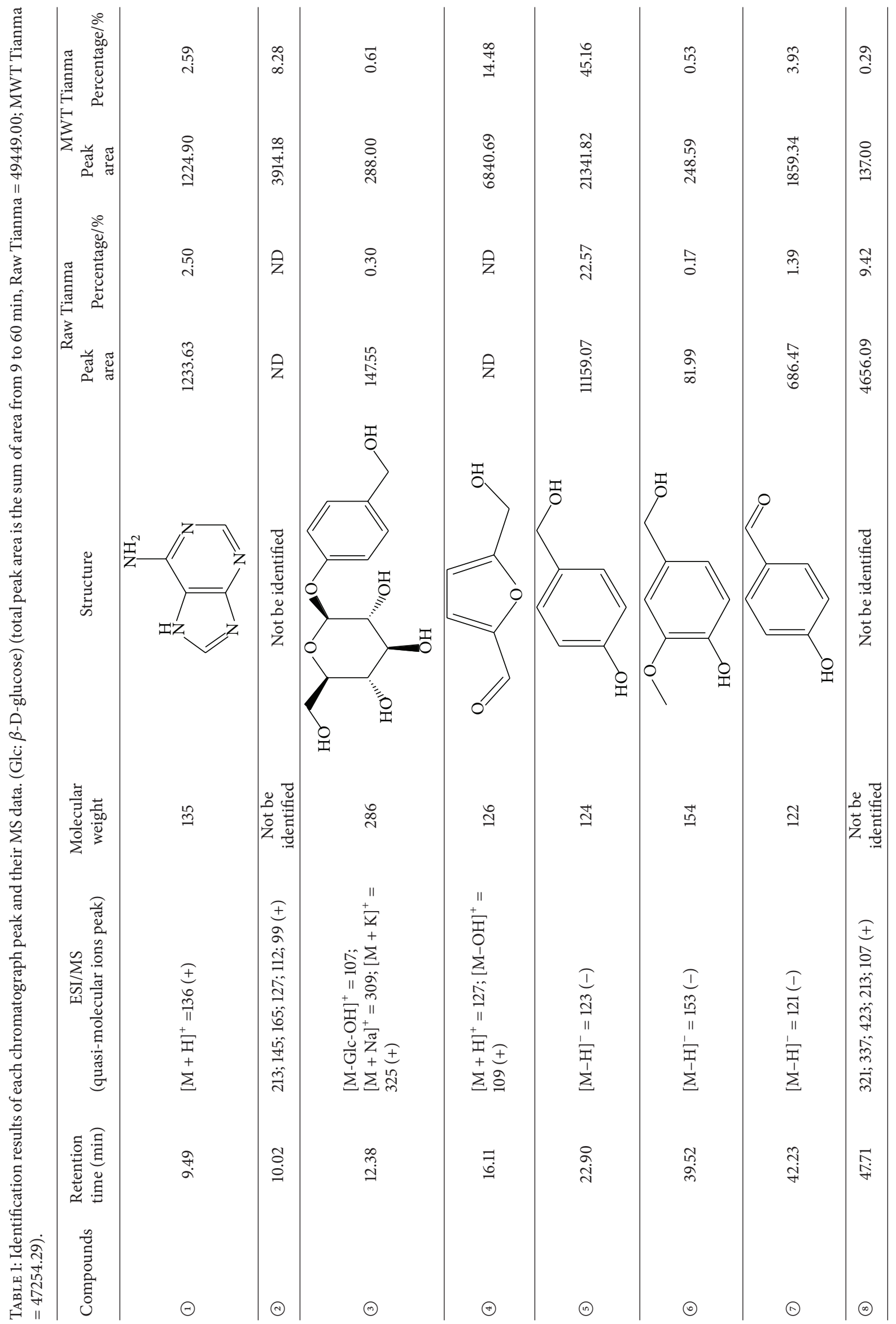




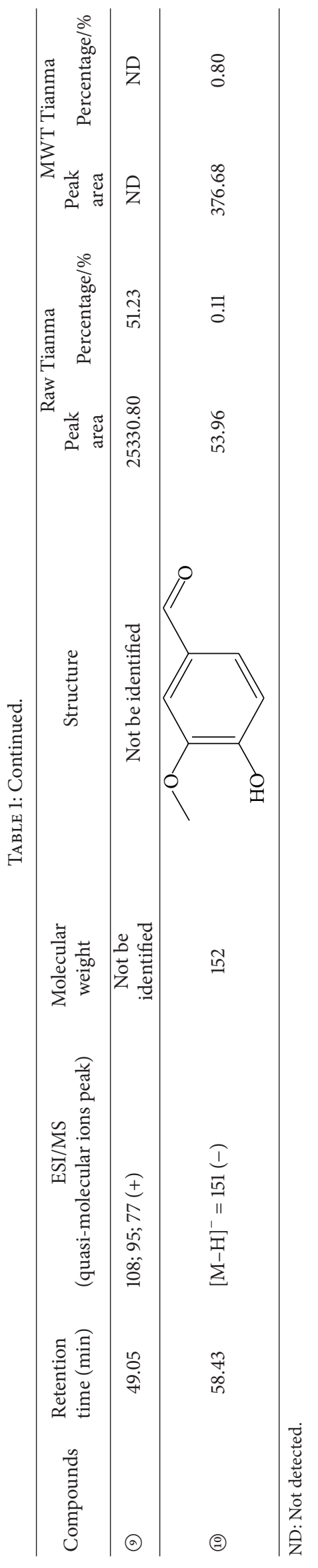




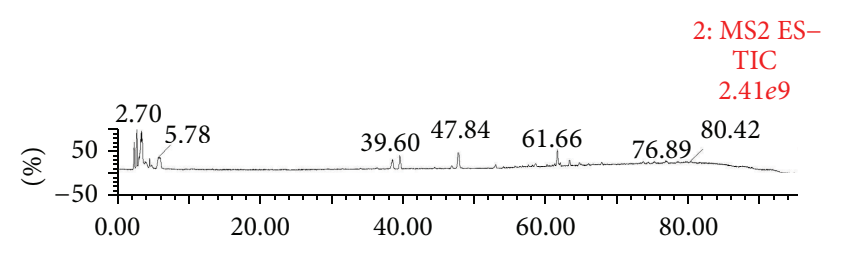

(a)
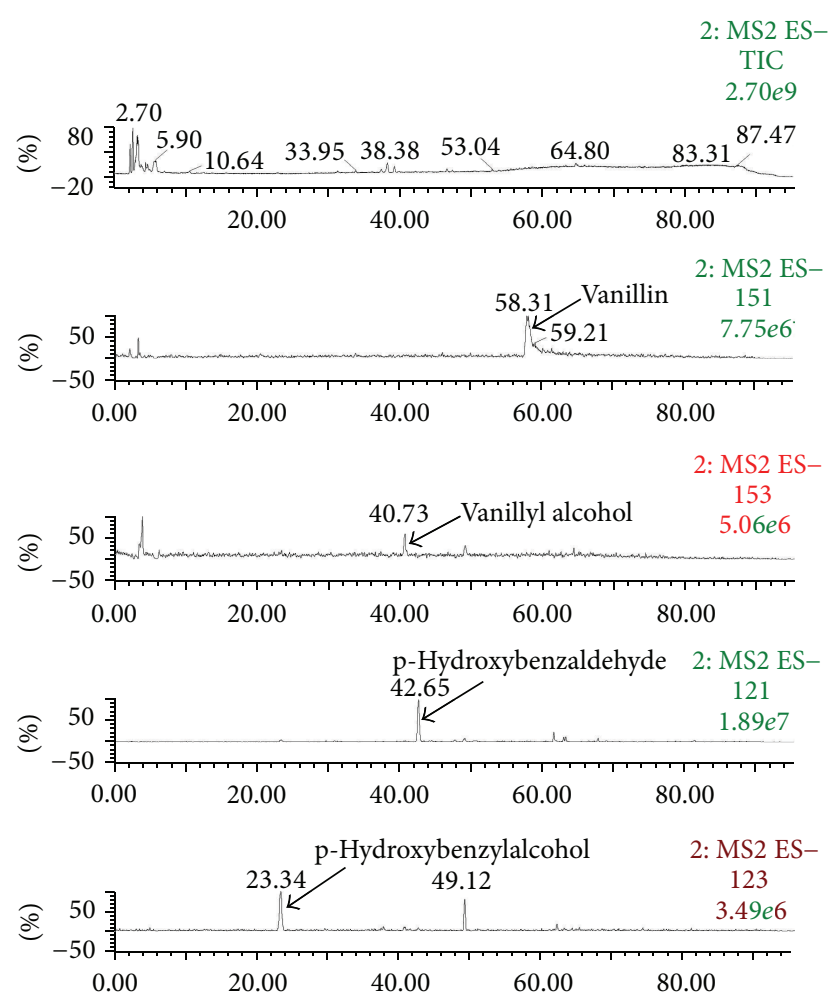

(b)

FIGURE 3: Total ion chromatogram ((a) raw Tianma extract; (b) MWT Tianma extract) and extracted ion chromatograms for representative phenolics of Tianma with no MWT.

might be 212 and 384 . Further research is needed to identify the exact chemical structure of these uncertain compounds using NMR spectroscopy, IR spectroscopy, and other higherend research tools after isolation and purification from raw Tianma and MWT Tianma.

3.2. The Possible Chemical Reactions under MWT. From Table 1, after the effect of microwave, contents of components (2), (3), (4), (5), (6), and (7) significantly increased and contents of components (8) and (9) significantly decreased. As an important evaluating index to reflecting the quality of Tianma, the contents of (3)gastrodin and (5)gastrodigenin (p-hydroxybenzylalcohol) in MWT Tianma, especially, were both twice as much as those in raw Tianma. Thus, microwave might be thought of as a powerful new technology to process fresh Tianma into corresponding products with much more effective components. What is more, components (2) and
(4) probably are MWT converted products. The possible chemical reaction pathways are elucidated in Figure 7.

In Figure 7, compounds A, B, C, and D represent four classes of compounds containing similar structure with p-hydroxylbenzylalcohol, p-hydroxybenzaldehyde, vanillyl alcohol, and vanillin, respectively. For instance, compound A containing two or more p-hydroxylbenzylalcohol moieties has been reported [29-31], and through the effect of microwave, compound A can be converted into phydroxylbenzylalcohol. Gastrodin is synthesized by the dehydration of p-hydroxylbenzylalcohol and glucose, and it is possible that gastrodin also may be converted from some compounds, such as parishins A, B, C, D, and E [29, $30,32]$, containing a similar structure to gastrodin. 5Hydroxymethylfurfural is a degradation product of sugars and is conversion mainly from glucose and fructose [33].

\section{Conclusions}

In this study, the effect of microwave treatment on fresh Tianma, which was evaluated by changes of several main components, was reported. As an important evaluating index to reflecting the quality of Tianma, the contents of gastrodin and gastrodigenin (p-hydroxybenzylalcohol) in MWT Tianma, especially, were both twice as much as those in raw Tianma, which shows that microwave treated fresh Tianma might be helpful in designing the processing of traditional Chinese medicine. The mechanism of microware processing, however, has not been understood yet. The effect of microwave should be a combination of thermal effect, nonthermal effect, natural catalyst existing in Tianma, and some other factors. Therefore, the application of microwave technology in traditional Chinese medicine needs to be researched further in the future.

\section{Abbreviation \\ MWT: Microwave treatment \\ TCM: Traditional Chinese medicine \\ MW: Molecular weight.}

\section{Conflict of Interests}

The authors declare that there is no conflict of interests.

\section{Acknowledgments}

The authors gratefully acknowledge the financial support of Science \& Technology Innovation Team of Chinese Traditional Medicine of Universities of Yunnan Province, the National Key Technology RD Program China (2011BAD46B00), Yunnan Science \& Technology Program (2011AB006), and Program of Traditional Chinese Pharmaceutical Science \& Technology Innovation Team of Yunnan Universities. 

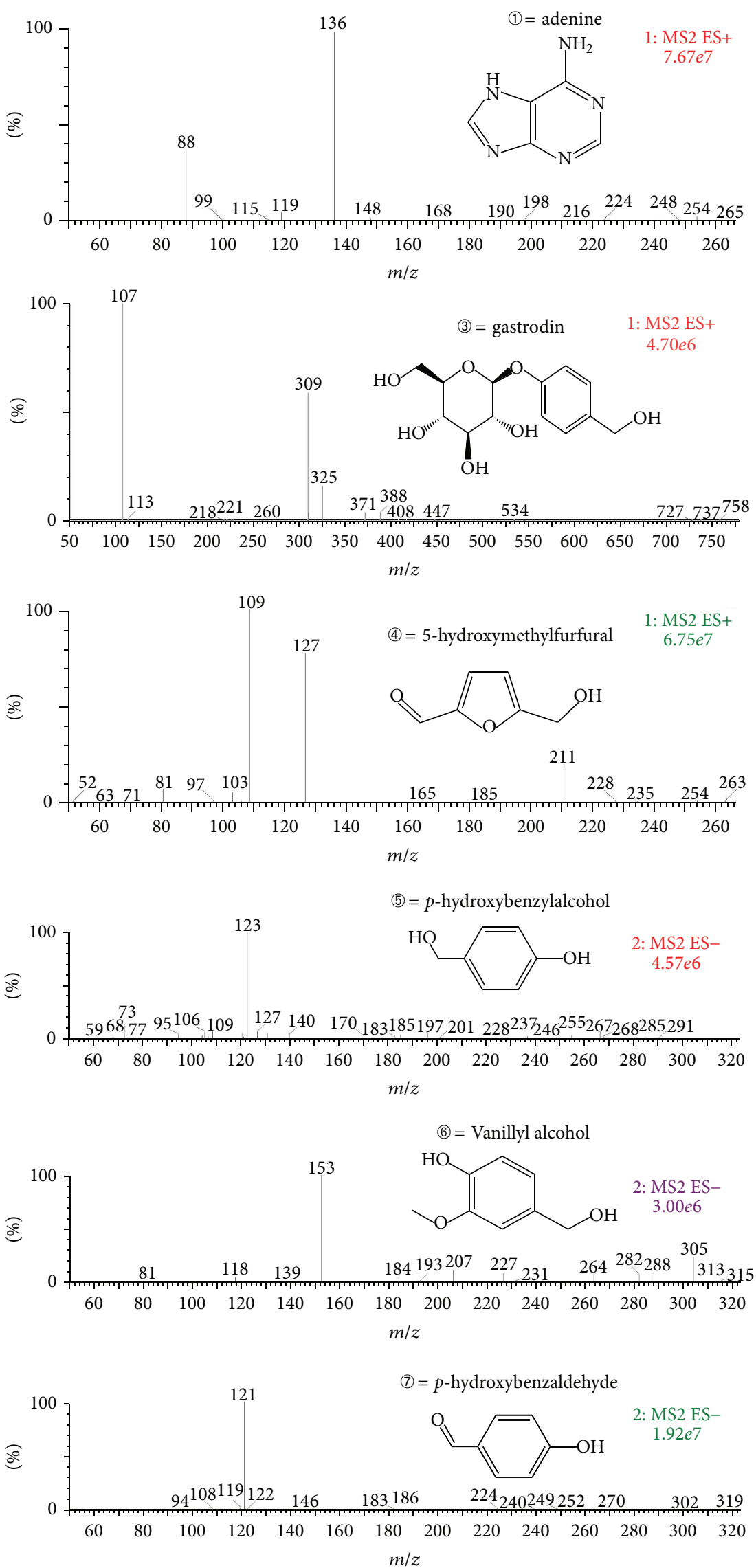

FIgURE 4: Continued. 


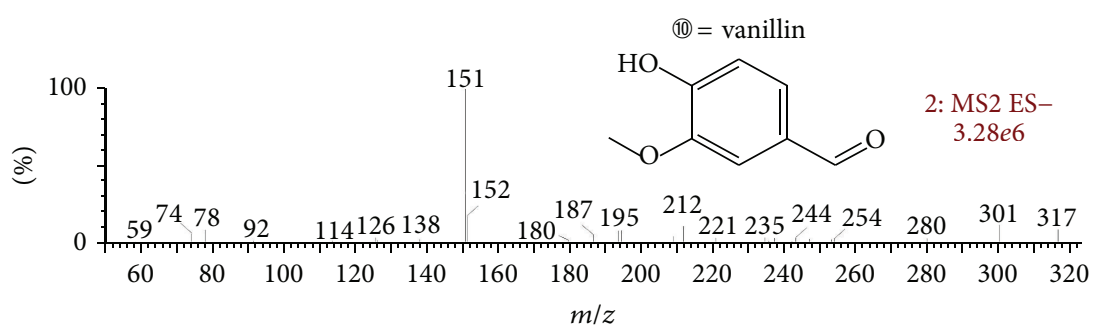

FIGURE 4: Representative MS spectra of (1)adenine, (3)gastrodin, (4)5-hydroxymethylfurfural, (5)p-hydroxybenzylalcohol, (6)vanillyl alcohol, (7)p-hydroxybenzaldehyde, and (10)vanillin.
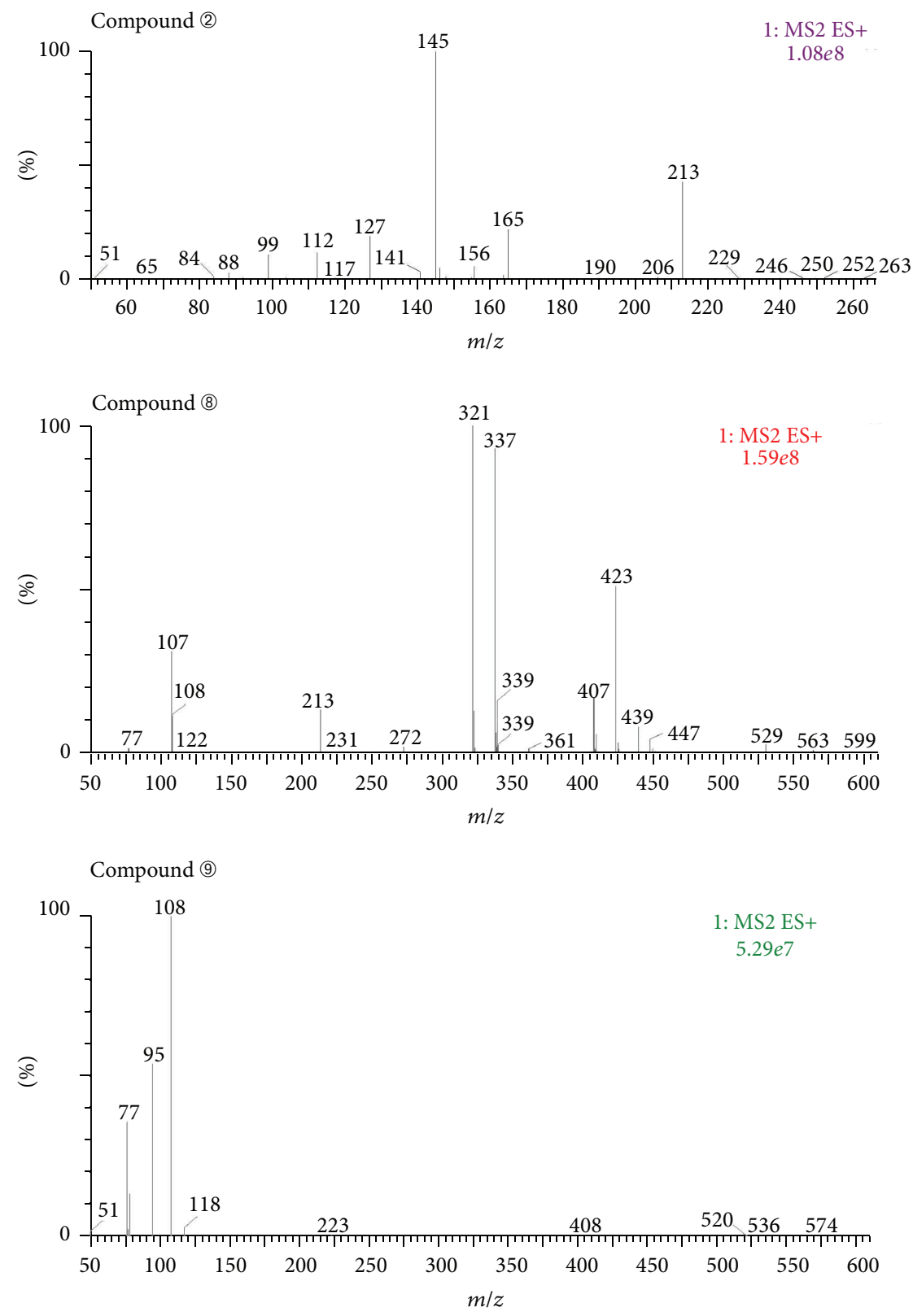

FIGURE 5: Representative MS spectra of the unknown compounds (2), (8), and (9. 
(1) Compound (9)

Possible molecular weight $=107$

Possible molecular formula $=\mathrm{C}_{7} \mathrm{H}_{9} \mathrm{~N}$

Possible structure a: $\mathrm{N}$-methylaniline

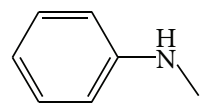

Possible structure b: o-toluidine or p-toluidine or $m$-toluidine

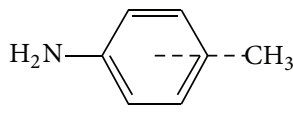

(2) Compounds (2) and (8)

Possible molecular weight of compound (2) $\left(\mathrm{M}_{(}\right)=212$

Possible molecular weight of compound (8) $\left(\mathrm{M}_{\odot}\right)=384$
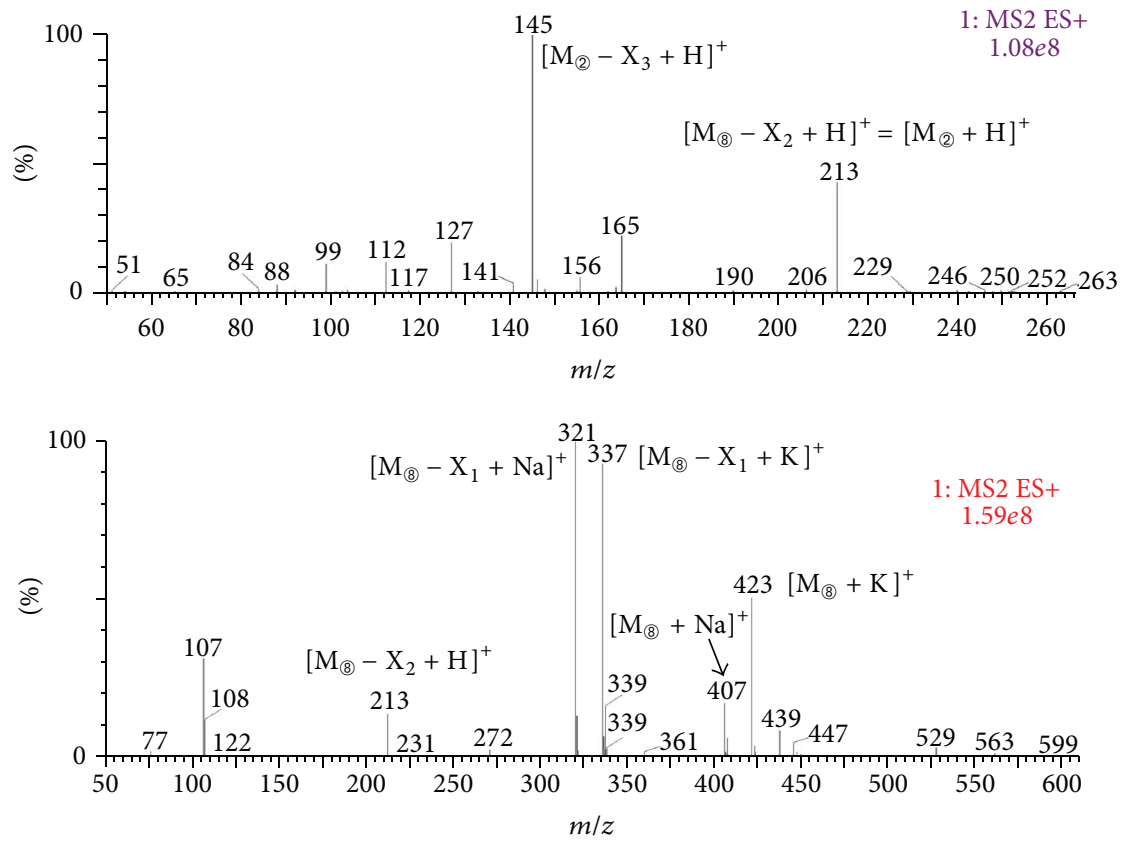

FigurE 6: The analysis results of the unknown compounds (2), (8), and (9).

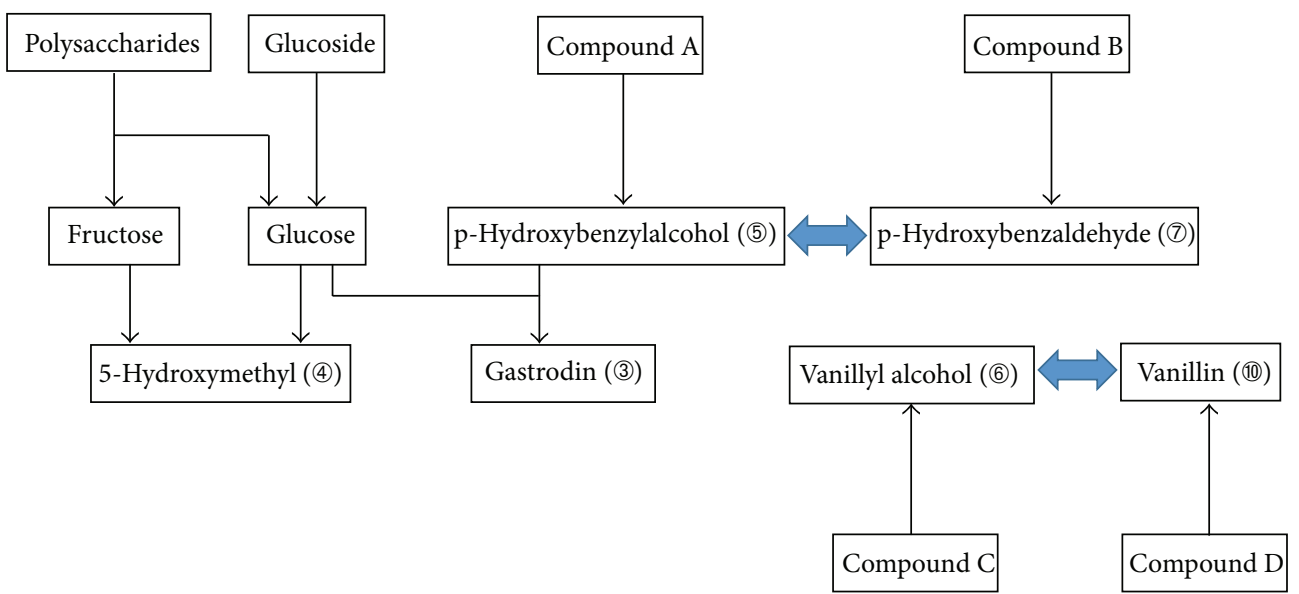

Figure 7: The possible chemical reaction pathways in Tianma under MWT. 


\section{References}

[1] S. Chow and S. Chen, "Notes on Chinese gastradia," Acta Botanica Yunnanica, vol. 5, pp. 361-368, 1983.

[2] S. P. Committee, Pharmacopoeia of the People's Republic of China, People's Medical Publishing House, Beijing, China, 2010.

[3] C. L. Liu, M. C. Liu, and P. L. Zhu, "Determination of gastrodin, p-hydroxybenzylalcohol, vanillyl alcohol p-hydroxylbenzaldehyde and vanillin in tall gastrodia tuber by high performance liquid chromatography," Chromatographia, vol. 55, no. 5-6, pp. 317-320, 2002.

[4] W. Tang and G. Eisenbrand, "Chinese drugs of plant origin; 1992," in Chemistry, Pharmacology, and Use in Traditional and Modern Medicine, pp. 97-103, 2000.

[5] S. F. Adam and H. Packard, Microwave Theory and Applications, Prentice-Hall, Upper Saddle River, NJ, USA, 1969.

[6] T. Takeuchi, C. Pereira, M. Braga, M. Maróstica Jr., P. Leal, and M. Meireles, "Low-pressure solvent extraction (solid-liquid extraction, microwave assisted, and ultrasound assisted) from condimentary plants," in Extracting Bioactive Compounds for Food Products, pp. 137-218, CRC Press, 2009.

[7] A. De La Hoz, Á. Díaz-Ortiz, and A. Moreno, "Microwaves in organic synthesis. Thermal and non-thermal microwave effects," Chemical Society Reviews, vol. 34, no. 2, pp. 164-178, 2005.

[8] J. A. Tanner, C. Romero-Sierra, and S. J. Davie, "Non-thermal effects of microwave radiation on birds," Nature, vol. 216, no. 5120, p. 1139, 1967.

[9] J. Jacob, L. H. L. Chia, and F. Y. C. Boey, "Thermal and nonthermal interaction of microwave radiation with materials," Journal of Materials Science, vol. 30, no. 21, pp. 5321-5327, 1995.

[10] A. Shazman, S. Mizrahi, U. Cogan, and E. Shimoni, "Examining for possible non-thermal effects during heating in a microwave oven," Food Chemistry, vol. 103, no. 2, pp. 444-453, 2007.

[11] R. J. Giguere, T. L. Bray, S. M. Duncan, and G. Majetich, "Application of commercial microwave ovens to organic synthesis," Tetrahedron Letters, vol. 27, no. 41, pp. 4945-4948, 1986.

[12] L. Perreux and A. Loupy, "A tentative rationalization of microwave effects in organic synthesis according to the reaction medium, and mechanistic considerations," Tetrahedron, vol. 57, no. 45, pp. 9199-9223, 2001.

[13] J. D. Moseley and C. O. Kappe, "A critical assessment of the greenness and energy efficiency of microwave-assisted organic synthesis," Green Chemistry, vol. 13, no. 4, pp. 794-806, 2011.

[14] A. E. Drouzas and H. Schubert, "Microwave application in vacuum drying of fruits," Journal of Food Engineering, vol. 28, no. 2, pp. 203-209, 1996.

[15] M. Zhang, J. Tang, A. S. Mujumdar, and S. Wang, "Trends in microwave-related drying of fruits and vegetables," Trends in Food Science \& Technology, vol. 17, no. 10, pp. 524-534, 2006.

[16] Z. Li, G. S. V. Raghavan, and V. Orsat, "Temperature and power control in microwave drying," Journal of Food Engineering, vol. 97, no. 4, pp. 478-483, 2010.

[17] V. Camel, "Recent extraction techniques for solid matricessupercritical fluid extraction, pressurized fluid extraction and microwave-assisted extraction: their potential and pitfalls," Analyst, vol. 126, no. 7, pp. 1182-1193, 2001.

[18] T. S. Ballard, P. Mallikarjunan, K. Zhou, and S. O’Keefe, "Microwave-assisted extraction of phenolic antioxidant compounds from peanut skins," Food Chemistry, vol. 120, no. 4, pp. 11851192, 2010.
[19] P. Wang, Q. Zhang, Y. Wang et al., "Evaluation of Soxhlet extraction, accelerated solvent extraction and microwave-assisted extraction for the determination of polychlorinated biphenyls and polybrominated diphenyl ethers in soil and fish samples," Analytica Chimica Acta, vol. 663, no. 1, pp. 43-48, 2010.

[20] G. A. Cardoso-Ugarte, G. P. Juárez-Becerra, M. E. Sosa Morales, and A. López-Malo, "Microwave-assisted extraction of essential oils from herbs," Journal of Microwave Power Electromagnetic Energy, vol. 47, no. 1, pp. 63-67, 2013.

[21] S. Ashraf, S. M. G. Saeed, S. A. Sayeed, and R. Ali, "Impact of microwave treatment on the functionality of cereals and legumes," International Journal of Agriculture and Biology, vol. 14, no. 3, pp. 365-370, 2012.

[22] W. Routray and V. Orsat, "Microwave-assisted extraction of flavonoids: a review," Food and Bioprocess Technology, vol. 5, no. 2, pp. 409-424, 2012.

[23] J. Kaasova, P. Kadlec, Z. Bubnik, B. Hubackova, and J. Prihoda, "Physical and chemical changes during microwave drying of rice," Chemical Papers-slovak Academy of Sciences, vol. 56, no. 1, pp. 32-35, 2002.

[24] W. Tochampa, N. Jittrepotch, T. Kongbangkerd, K. Kraboun, and K. Rojsuntornkitti, "The study of microwave heating time on chemical and microbiological properties and sensory evaluation in sweet fermented glutinous rice (Khao-Mark)," International Food Research Journal, vol. 18, no. 1, pp. 239-248, 2011.

[25] Y. Zhao, Q.-E. Cao, Y. Xiang, and Z. Hu, "Identification and determination of active components in Gastrodia elata Bl. by capillary electrophoresis," Journal of Chromatography A, vol. 849, no. 1, pp. 277-283, 1999.

[26] W. Li, H. Xiao, J. Hu, J. Wang, and X. Liang, "Study of phydroxyphenylic compounds in gastrodia elata blume by high performance liquid chromatography-electrospray-mass spectrometry," Chinese Journal of Analytical Chemistry, vol. 31, no. 8, pp. 954-957, 2003.

[27] X. L. Xia, M. F. Xia, H. J. Yang, L. Zhang, B. Zhang, and W. Wang, "LC-MS/MS analysis of purine content in light Chinese rice wine," Modern Food Science \& Technology, vol. 26, no. 12, pp. 1399-1402, 2010.

[28] M. Aziz, J. M. He, J. H. Sun, J. F. Bai, and A. Zeper, "HPLC-MS/MS determination of the content of 5-hydroxymethylfurfural in Chinese traditional medicine injection," Chinese Journal of Pharmaceutical Analysis, vol. 30, no. 7, pp. 1195$1198,2010$.

[29] H. Taguchi, I. Yosioka, K. Yamasaki, and I. H. Kim, "Studies on the constituents of Gastrodia elata Blume," Chemical and Pharmaceutical Bulletin, vol. 29, no. 1, pp. 55-62, 1981.

[30] J.-H. Lin, Y.-C. Liu, J.-P. Hau, and K.-C. Wen, "Parishins B and C from rhizomes of Gastrodia elata," Phytochemistry, vol. 42, no. 2, pp. 549-551, 1996.

[31] J. Hayashi, T. Sekine, S. Deguchi et al., "Phenolic compounds from Gastrodia rhizome and relaxant effects of related compounds on isolated smooth muscle preparation," Phytochemistry, vol. 59, no. 5, pp. 513-519, 2002.

[32] X.-D. Yang, J. Zhu, R. Yang, J.-P. Liu, L. Li, and H.-B. Zhang, "Phenolic constituents from the rhizomes of Gastrodia elata" Natural Product Research, vol. 21, no. 2, pp. 180-186, 2007.

[33] X. Qi, M. Watanabe, T. M. Aida, and R. L. Smith Jr., "Catalytical conversion of fructose and glucose into 5-hydroxymethylfurfural in hot compressed water by microwave heating," Catalysis Communications, vol. 9, no. 13, pp. 2244-2249, 2008. 

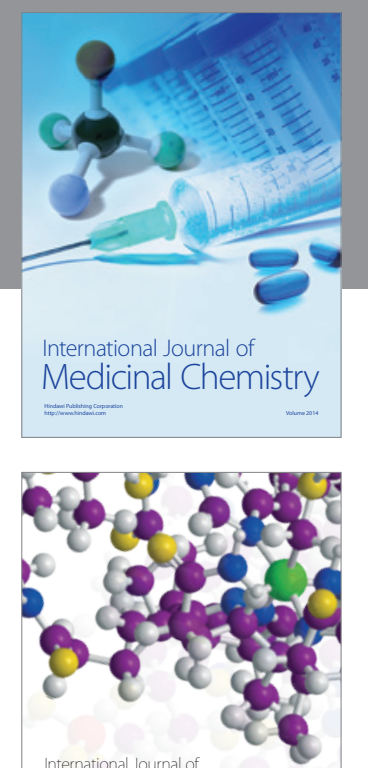

\section{Carbohydrate} Chemistry

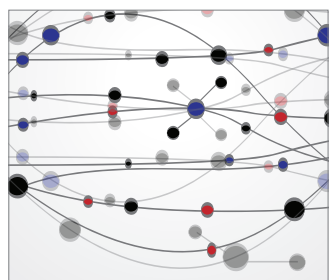

The Scientific World Journal
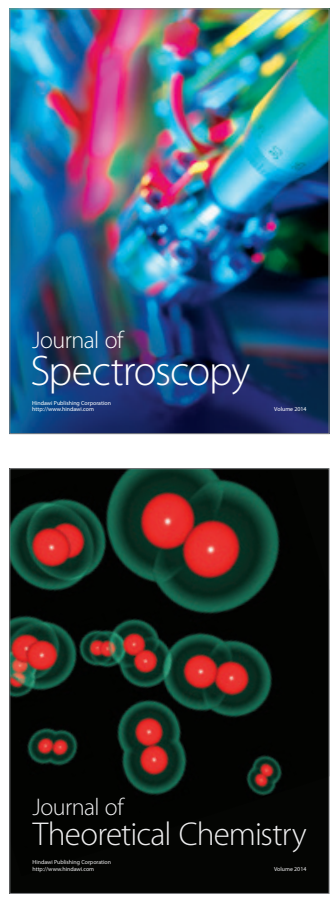
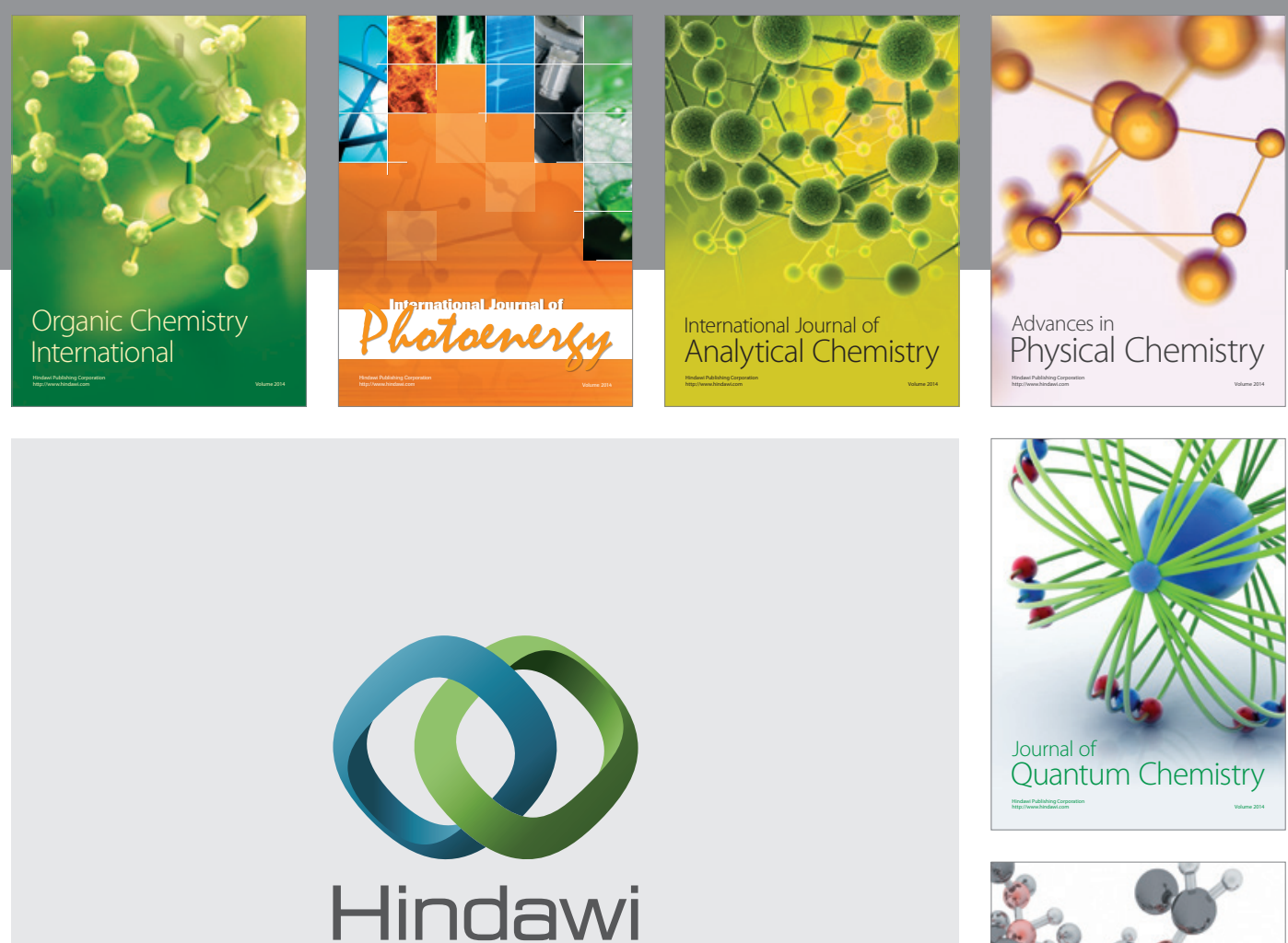

Submit your manuscripts at

http://www.hindawi.com

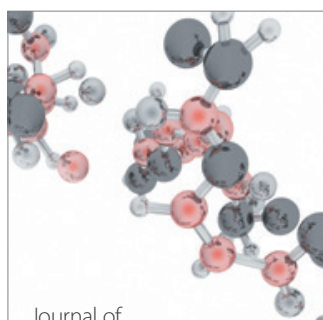

Analytical Methods

in Chemistry

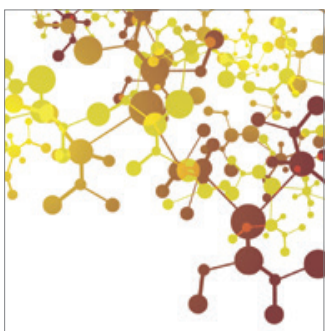

Journal of

Applied Chemistry

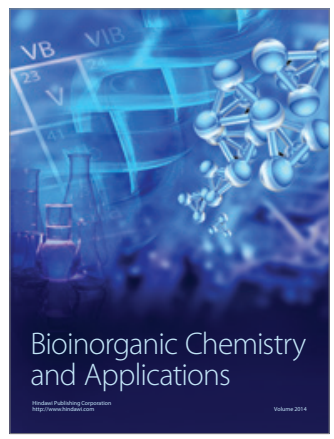

Inorganic Chemistry
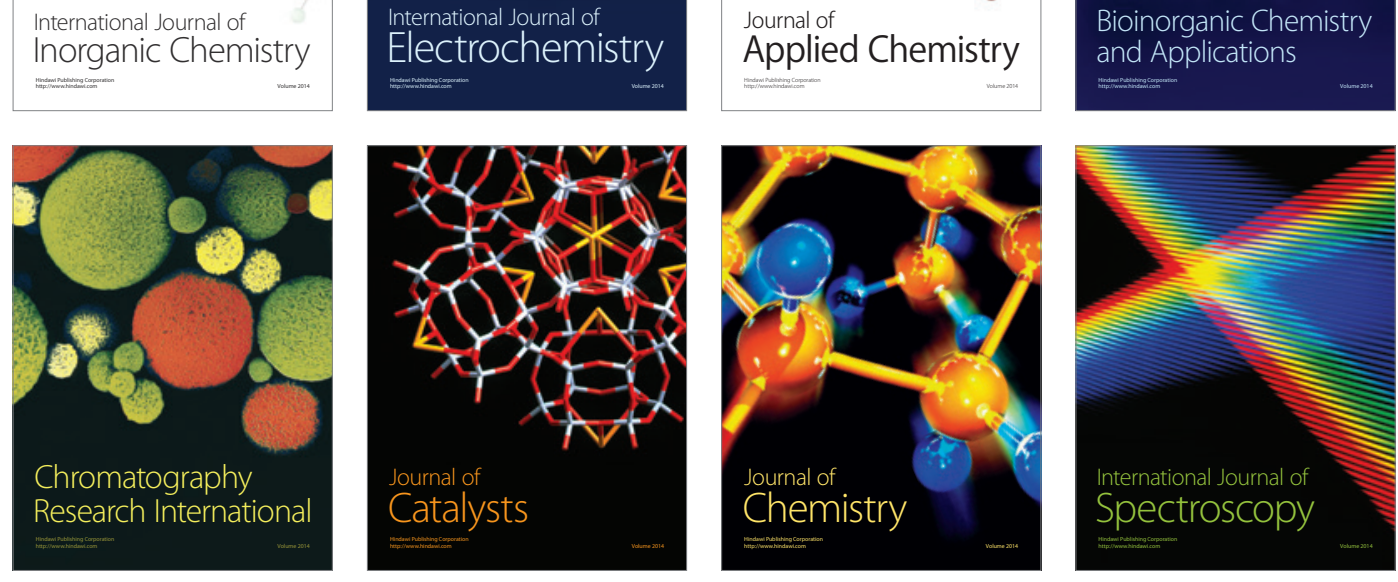